\title{
Topological photonics: From crystals to particles
}

\author{
Gleb Siroki, ${ }^{1, *}$ Paloma A. Huidobro, ${ }^{1}$ and Vincenzo Giannini ${ }^{1,2, \dagger}$ \\ ${ }^{I}$ Department of Physics, Imperial College London, London SW7 2AZ, United Kingdom \\ ${ }^{2}$ Instituto de Estructura de la Materia, IEM-CSIC, E-28006 Madrid, Spain \\ (Received 7 April 2017; revised manuscript received 5 July 2017; published 25 July 2017)
}

\begin{abstract}
Photonic crystal topological insulators host protected states at their edges. In the band structure these edge states appear as continuous bands crossing the photonic band gap. They allow light to propagate unidirectionally and without scattering. In practice it is essential to make devices relying on these effects as miniature as possible. Here we study photonic topological insulator particles (finite crystals). In such particles the edge state frequencies are discrete. Nevertheless, the discrete states support pseudospin-dependent unidirectional propagation. They allow light to bend around sharp corners similarly to the continuous edge states and act as topologically protected whispering gallery modes, which can store and filter light as well as manipulate its angular momentum. Though we consider a particular all-dielectric realization that does not require a magnetic field, the results in the findings are general, explaining multiple experimental observations of discrete transmission peaks in photonic topological insulators.
\end{abstract}

DOI: 10.1103/PhysRevB.96.041408

Introduction. The invention of photonic crystals has enriched the field of photonics [1,2]. More recently the field benefited from applying the ideas of topology to photonic band structures [3]. This was triggered by the discovery [4] and experimental confirmation $[5,6]$ of electronic topological insulators (TIs). The hallmark of TIs are protected states occurring at the boundary of their crystals [Fig. 1(a)]. These states support unidirectional propagation and are immune to certain defects. Their analogs are also being explored in acoustics [7-9], optical lattices [10], plasmonics [11-14], and especially photonics [15]. While photonic crystal analogs of a three-dimensional (3D) TI have only been recently proposed $[16,17]$, their two-dimensional (2D) counterparts have already been realized experimentally $[15,18-23]$. Some of these realizations employ magnetic fields $[18,20]$ while others preserve the time-reversal symmetry [21-24]. In the latter the angular momentum of light (spin) provides an additional degree of freedom [25-27]. The spin can be used within spin-chiral optical networks and spin-controlled gates [28,29]. In contrast to conventional optical devices, these will be more robust to manufacturing imperfections due to the absence of spin degeneracy. The latter is especially true for TIs involving magnetic fields where a single edge band can exist $[18,20]$.

Here we study a photonic TI particle (finite crystal) made of dielectric rods [30-32] as proposed by $\mathrm{Wu}$ and $\mathrm{Hu}$ [30] which does not require an external magnetic field and is completely scalable. As opposed to an infinite photonic TI [Fig. 1(a)] the particle has a discrete rather than continuous spectrum of edge states, as shown in Fig. 1(b). These states exist for particles of different shapes, being a topologically protected version of whispering gallery modes [33]. In contrast to ordinary microcavities, they are not pseudospin degenerate and support unidirectional propagation which gradually reduces with disorder.

While we study a particular photonic crystal, our main findings are general and hold for other realizations of photonic

\footnotetext{
*gleb.siroki14@imperial.ac.uk

${ }^{\dagger}$ www.gianninilab.com
}

TIs. They explain the discrete peaks observed in transmission $[18,20-23]$ unexplained previously. These occur because the photonic crystals considered in experiments contain at $O\left(10^{3}\right)$ artificial atoms, c.f. electronic TIs containing $O\left(10^{23}\right)$ atoms [34]. Thus photonic TIs are often accurately described as particles whose discrete spectrum gives rise to the peaks in transmission.

Edge states of the photonic TI particle. The photonic TI considered consists of dielectric rods [Fig. 1(c)] extending along the $z$ axis. Six of these rods constitute an artificial atom [30] as shown in Fig. 1(d). A photonic crystal made of these atoms arranged on a triangular lattice possesses $C_{6}$ and timereversal symmetries. Adjusting the atomic size allows one to achieve a topological phase for TM polarization [30]. Each atom carries four orbitals labeled $p_{ \pm}=p_{x} \pm i p_{y}$ and $d_{ \pm}=$ $d_{x^{2}-y^{2}} \pm i d_{x y}$ according to their $E_{z}$ fields. Using symmetry arguments a photonic $4 \times 4 k \cdot p$ Hamiltonian can be found [30], similar to the electronic Bernevig-Hughes-Zhang (BHZ) Hamiltonian [35] (see Supplemental Material [36]). We apply the method of Imura et al. [37] to the TI particle with a circular cross section lying in the $x-y$ plane. The two solutions,

$$
\begin{aligned}
& \mathbf{c}_{+, m}=R(r)\left(e^{-i \phi}, 1,0,0\right)^{T} e^{i m \phi} / \sqrt{2}, \\
& \mathbf{c}_{-, m}=R(r)\left(0,0, e^{i \phi}, 1\right)^{T} e^{i m \phi} / \sqrt{2},
\end{aligned}
$$

are the coefficients in front of $p_{+}, d_{+}, p_{-}, d_{-}$in the field expansion [Eq. (10) of Supplemental Material [36]]. The azimuthal number, $m=0, \pm 1, \pm 2 \ldots$, ensures $c_{ \pm, m}(2 \pi+\phi)=$ $c_{ \pm, m}(\phi)$. The function $R(r)$ decays exponentially away from the boundary of the particle. The state frequencies are given by

$$
w_{ \pm, m}=w_{0}+\frac{C}{r_{\mathrm{P}}}\left(\frac{1}{2} \pm m\right),
$$

where $w_{0}$ is in the middle of the band gap, $r_{\mathrm{P}}$ is the particle radius, and $C$ is some constant. Thus the states come in equally spaced pairs with $(+, m)$ and $(-,-m)$ being degenerate. This is analogous to the pairs of edge states in electronic TI nanoparticles that exist due to time-reversal symmetry of the 
(a)

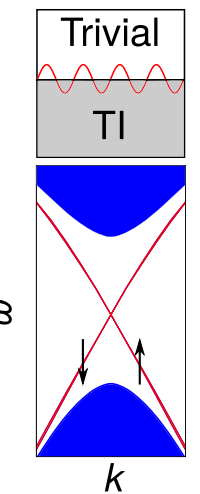

(b)

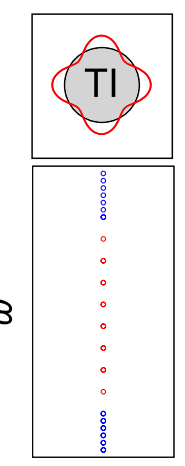

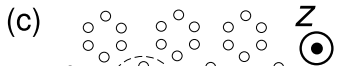

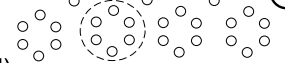

(d)

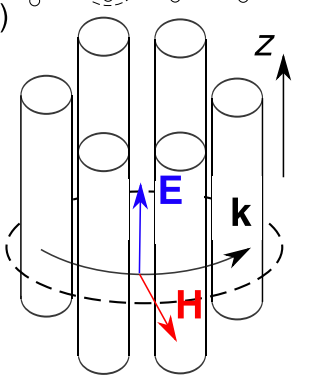

FIG. 1. (a) Top panel: An edge state of a photonic TI crystal. Bottom panel: The crystal band structure with blue regions representing the bulk bands. Red bands inside the bulk band gap are the topologically protected edge states. (b) Spectrum of a photonic TI particle, where the red circles represent edge states that reside inside the bulk gap. (c) Top view of the photonic TI used here. (d) Six dielectric rods arranged in a hexagon make up an artificial atom (encircled).
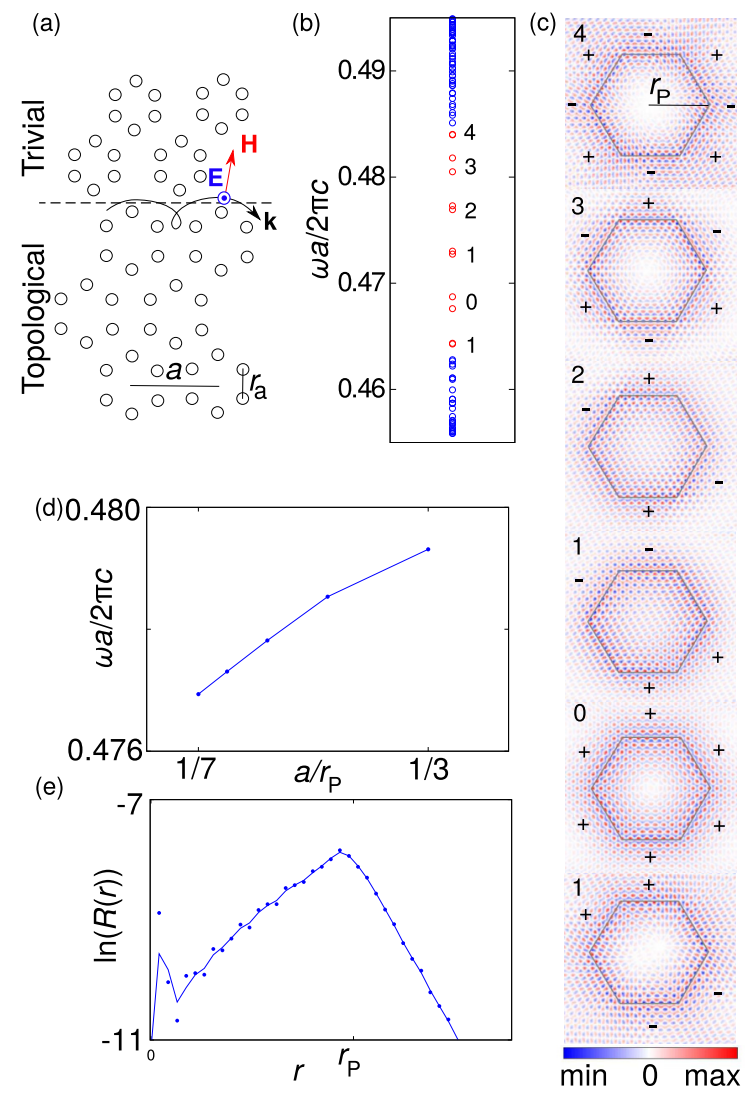

FIG. 2. (a) Interface between topological and trivial photonic crystals supports protected edge states. (b) Frequency spectrum of a hexagonal photonic TI particle (169 atoms) embedded in trivial matrix contains six pairs of TM edge states. They are labeled by the number of nodes in azimuthal direction. (c) $E_{z}$ fields for the states are shown (one state from each pair); + and - denote the relative phase of the field. (d) The frequency of the state labeled 2 decreases linearly with the particle radius. (e) The $E_{z}$ field averaged over azimuthal angle for the state labeled 2. The resulting radial distribution peaks close to the edge and decays exponentially away from it.

Schrodinger equation [38,39]. However, because photons are not fermions, one needs another symmetry to realize a TI. Here this is realized by the $C_{6}$ symmetry of the photonic TI crystal [30]. The importance of the crystal symmetry becomes apparent below.

We have performed numerical simulations for a hexagonal particle, choosing the rods to be made of silicon $(\epsilon=11.7)$ with air in between and other parameters as in Ref. [30]. We simulate the hexagonal TI particle of radius $r_{\mathrm{P}} / a=7$ (169 atoms), where $a$ is the distance between atoms. The particle is encased in a trivial matrix as shown schematically in Fig. 1(b). The edge states appear at the boundary between the topological $\left(r_{\mathrm{a}}>a / 3\right)$ and trivial atoms $\left(r_{\mathrm{a}}<a / 3\right)$ as illustrated in Fig. 2(a). Their spectrum, obtained with frequency domain simulations using the MPB package [40], is shown in Fig. 2(b). There are six pairs of states, half of which are shown in Fig. 2(c). The $E_{z}$ fields for the remaining six states and the full supercell (not shown) can be found in the Supplemental Material [36]. Each state is a standing wave made up of two counterpropagating waves, c.f. states at $\pm k$ in Fig. 1(a). These two waves have opposite pseudospin $\left(H_{x} \pm i H_{y}\right)$ as required by the time-reversal symmetry of Maxwell equations and each supports unidirectional propagation of light, as shown below. The standing waves themselves come in pairs with approximately equal spacing, agreeing with Eq. (3). The states are not exactly degenerate because the particle is not circular. Also, at its boundary the $C_{6}$ symmetry is only approximate

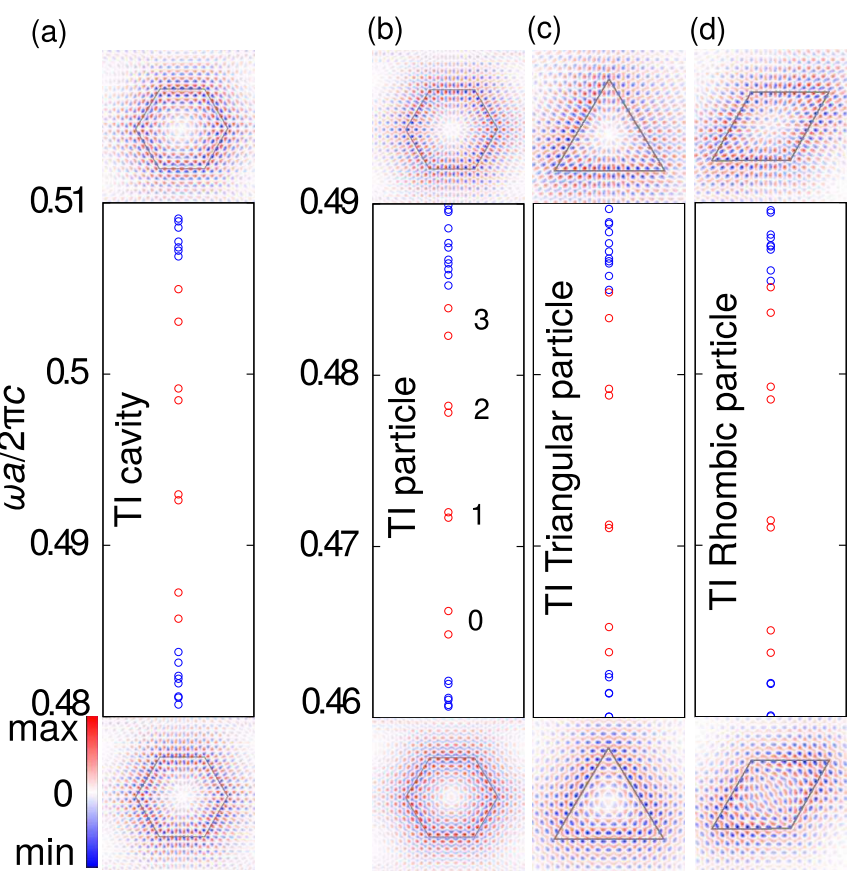

FIG. 3. The top (bottom) figure shows the $E_{z}$ field of the highest (lowest) edge state for (a) the "inverse" case of a trivial particle (91 atom) inside the topological matrix containing four pairs of TM edge states labeled by red circles. (b) A topological particle of the same size inside the trivial matrix. The field distributions are very similar and so are the spectra, except for a shift in frequency. (c), (d) Triangular and rhombic particles (66 and 64 atoms). The edge states exist for particles of different shapes and are able to bend around sharp corners. 
because of the mismatch between the topological and trivial atoms. They have similar radii so the effect is weak and further decreases radius. In contrast, the necessary symmetry (time reversal) is always present in electronic TI nanoparticles. There the states retain their degeneracy but get pushed out of the bulk gap as the size decreases [38,39]. For the photonic TI particle it is also true, and the number of edge states increases with $r_{\mathrm{P}}$ as implied by Eq. (3) and Fig. 2(d). As the particle becomes larger, additional pairs of edge states emerge from the bulk. For very small sizes they are completely pushed out of the photonic gap because there is not enough bulk to support them. The effect was predicted for ribbons of photonic TIs [30] and experimentally observed for slabs of electronic TIs [34]. This can be ascribed to the overlap of wave functions from opposite sides as a TI sample becomes small. The states themselves decay exponentially away from the edge, see Fig. 2(e). The number of azimuthal nodes they have agrees exactly between numerical and analytical results (see Supplemental Material [36]) - to obtain a state with no nodes in $E_{z}$ field we need coefficients $e^{\mp i \phi}\left(e^{\mp 2 i \phi}\right)$ in front of $p_{ \pm}\left(d_{ \pm}\right)$s.t. $E_{z}$ contains $p_{x} \cos \phi+p_{y} \sin \phi\left(d_{x^{2}-y^{2}} \cos 2 \phi+d_{x y} \sin 2 \phi\right)$. This ensures that the 'atomic' orbitals rotate with $\phi$ and occurs for the states $(+, m=-2)$ and $(-, m=2)$. These have frequency $w=w_{0}-3 C / 2 r_{\mathrm{P}}$ according to Eq. (3) agreeing with Fig. 2 $(b, c)$. The pair of states immediately above (below) has an

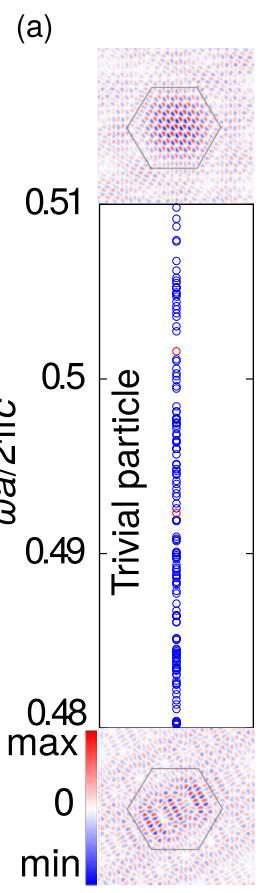

(b)

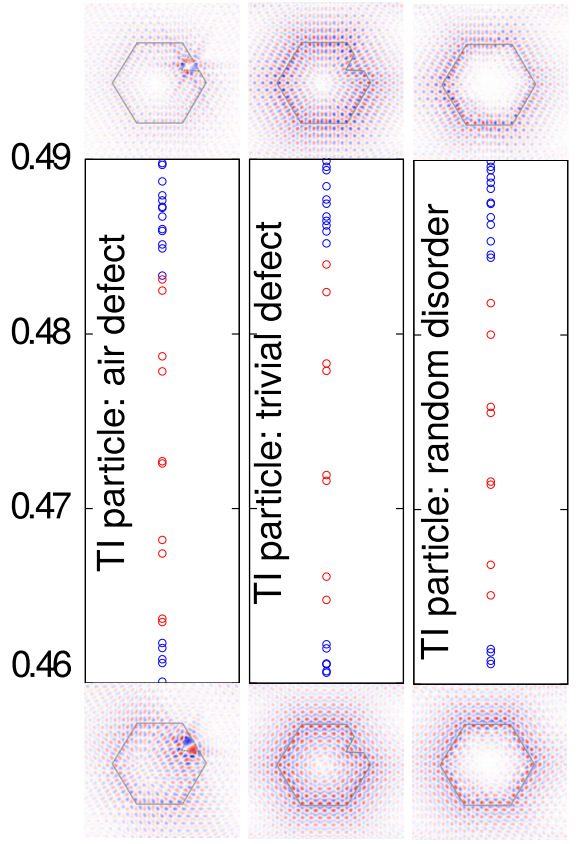

FIG. 4. The top (bottom) figure shows the $E_{z}$ field of the highest (lowest) state marked by a red circle for each particle (91 atoms). (a) A trivial particle inside the trivial matrix without edge states. (b) A TI particle inside the trivial matrix with three atoms removed. There is a 1:1 correspondence to unperturbed states with an additional pair emerging from the bulk. (c) Three topological atoms are replaced by trivial ones. (d) A TI particle with positional disorder. The rods in topological atoms are randomly moved by $10 \%$. This weakly perturbs the states, suggesting they will be robust against manufacturing imperfections. additional factor of $e^{ \pm i \phi}$ giving one node as Fig. 2(c) indeed shows. We stress that in electronic TIs the pairs occur due to time-reversal symmetry. In contrast, here the pairs occur due to the photonic crystal symmetry and each edge state (standing wave) is made of two time-reversed waves. The edge states also occur in pairs for particles of other shapes, as seen in Fig. 3. The particles are stronger and the states experience stronger local breaking of $C_{6}$ symmetry (see Supplemental Material [36]). The insensitivity to the shape illustrates their topological origin, c.f. the spectrum of a trivial particle in a trivial matrix shown in Fig. 4(a).

Photonic TI particle with a defect. The robustness against certain types of disorder is a distinct feature of topological states, however, it is only guaranteed as long as the necessary symmetries are preserved. Thus our photonic TI crystal is
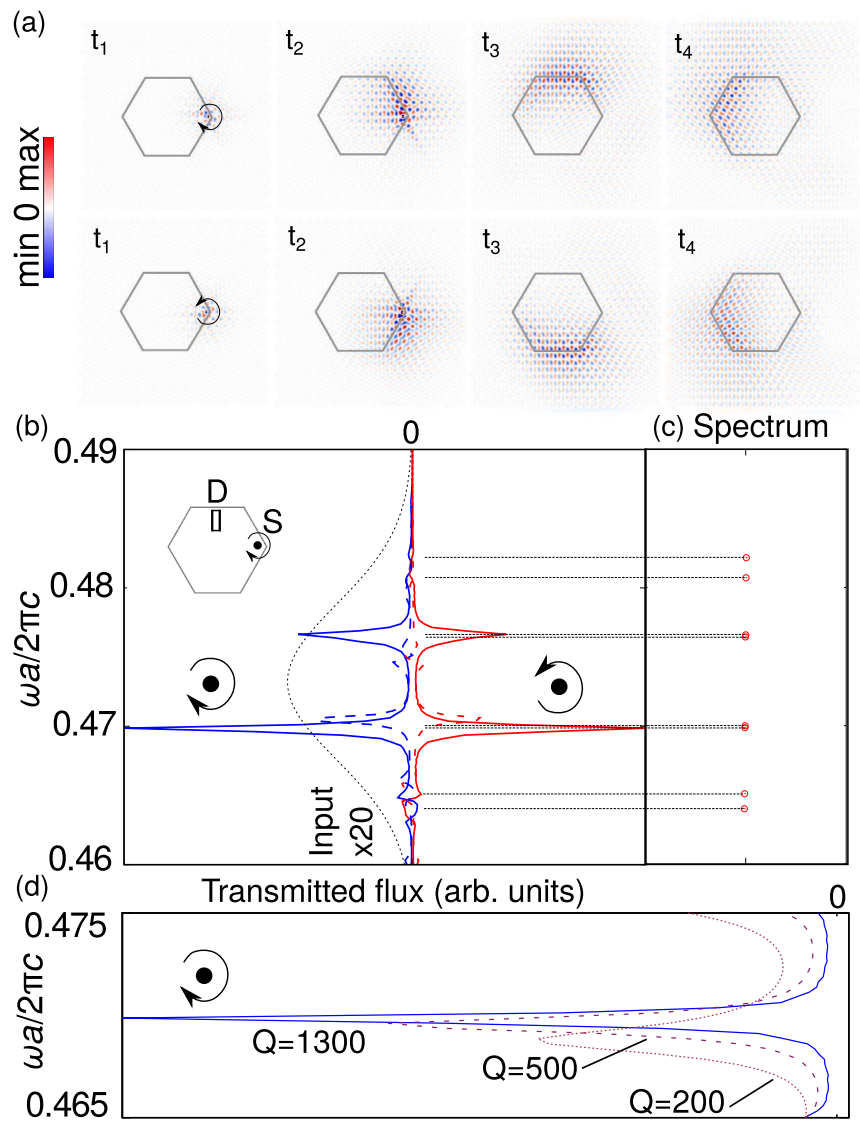

FIG. 5. Time-domain simulations of the photonic TI particle (91 atom) edge states. (a) $E_{z}$ field of a pulse travels in opposite directions depending on its pseudospin. (b) Inset: The particle, source (S), and detector (D). The transmission spectrum shows discrete peaks, positive for transmission in $x$ direction. Blue (red) solid lines correspond to $H_{x}+i H_{y}\left(H_{x}-i H_{y}\right)$ pseudospin. Dashed lines show corresponding results with $10 \%$ disorder in positions of rods constituting the particle (see main text). The black dotted line shows the profile of the input Gaussian pulse (magnified). (c) Horizontal lines show real parts of the state frequencies. (d) The width of the states depends on the thickness of the surrounding trivial matrix. Making the matrix two (four) trivial atoms thinner than above decreases the largest quality factor down to 500 (200), as indicated by the dashed (dotted) line. 
less robust against defects as it relies on the $C_{6}$ symmetry of the lattice. A strong symmetry breaking occurs when we completely remove three atoms from the TI particle as shown in Fig. 4(b). The spectrum contains an additional pair of edge states which has emerged from the bottom bulk band. Their field profiles can be matched to the unperturbed ones and the states support (and enhance) propagation in one direction (see Supplemental Material [36]). On the other hand, replacing the topological atoms with trivial ones has a weaker effect because the rods are less displaced from their ideal positions in the bulk topological crystal. Here the spectrum and the edge states are hardly affected, as seen in Fig. 4(c). This is because slightly different radii of topological and trivial atoms cause a weak $C_{6}$ symmetry breaking, as quantified in the Supplemental Material [36]. Finally, in practice the constituent rods can be displaced from their ideal positions. We have performed simulations for this case by displacing the rods in each topological atom randomly by $10 \%$. The results shown in Fig. 4(d) show that the states are weakly affected. The above results suggest that the states can be realized in practice. The crucial question to answer is whether they support unidirectional propagation.

Unidirectional propagation of light. For a semi-infinite crystal of the photonic TI considered, the slope of the continuous edge bands guarantees unidirectional propagation determined by the pseudospin $\left(H_{x} \pm i H_{y}\right)$, see Fig. 1(a). To show this for the present case of discrete states we used the finite-difference time-domain method as implemented in the MEEP package $[40,41]$. The TI particle is surrounded by the trivial matrix, and the simulation cell walls are covered with a perfectly matched layer (see Supplemental Material [36]). We excite the edge states with two TM-polarized dipoles $90^{\circ}$ out of phase with each other $\left(H_{x} \pm i H_{y}\right)$. They emit a Gaussian pulse whose pseudospin determines its direction of propagation as seen in Fig. 5(a). Moreover, the power transmission (normalized by the $Q$ factor) through a detector seen in Fig. 5(b) shows unidirectional propagation. The transmission spectrum confirms that each edge state (standing wave) consists of two counterpropagating waves as required by the time-reversal symmetry of Maxwell's equations. They propagate in opposite directions and either one or the other is excited, depending on the pseudospin of the incident light. In addition, we considered transmission for the particle with positional disorder as in Fig. 4(d). The resultant spectrum still shows unidirectional transmission for each given pseudospin, as seen in Fig. 5(b). The peaks are lower because the disorder increases scattering of the unidirectional wave into its degenerate partner with the opposite pseudospin. (For the effects of localized disorder, see Supplemental Material [36].) MEEP also allows one to obtain complex frequencies of the edge states. Their real parts, shown in Fig. 5(c), agree well with the frequency domain simulations in Fig. 3(b). The $Q$ factors are already $O\left(10^{3}\right)$ when the particle is surrounded by the trivial matrix, which is several atoms thick. Thinner layers lead to more leakage as well as $C_{6}$ symmetry breaking, and hence backscattering as is evident from Fig. 5(d). Finally, we stress that the unidirectional propagation for the photonic TI considered here has been recently observed as discrete transmission peaks [22,23]. In fact, the samples used in [18,20-23] have similar sizes to the particles studied here; therefore discrete states provide a natural interpretation for the peaks observed.

Conclusions. To summarize, we have investigated discrete edge states that emerge in a photonic TI particle. As an example we used a particular realization of a TI made of dielectric rods which does not require magnetic fields. The particle's spectrum contains edge states that occur in pairs. The spacing between the pairs decreases with the particle's size, tending to the continuum limit. Each edge state, in turn, is made up of two counterpropagating waves with opposite pseudospins. These edge states are insensitive to the particle's shape, as explicitly illustrated for hexagonal, rhombic, and triangular particles. They are robust against certain defects but disappear for very small particles where the wave functions from the opposite edges overlap, so one needs to ensure that the constructed photonic TI has enough bulk to support the edge states. Furthermore, the transmission frequencies can be adjusted with size using the relations found. The states support unidirectional propagation along the edge which can be switched with pseudospin. Such microcavity can be used to manipulate photons and can be arranged in arrays. Moreover, the edge states of TI particles will have a peculiar effect on the photonic local density of states and radiation of chiral molecules. Their behavior provides insight into the transport properties of electronic TI nanoparticles [38] where analogous first-principles calculations are impossible.

Acknowledgments. The authors thank Peter Haynes for useful discussions. G.S. was supported through a studentship in the Centre for Doctoral Training on Theory and Simulation of Materials at Imperial College London funded by EPSRC Grant No. EP/L015579/1.
[1] E. Yablonovitch, Phys. Rev. Lett. 58, 2059 (1987)

[2] S. John, Phys. Rev. Lett. 58, 2486 (1987).

[3] F. D. M. Haldane and S. Raghu, Phys. Rev. Lett. 100, 013904 (2008).

[4] C. L. Kane and E. J. Mele, Phys. Rev. Lett. 95, 146802 (2005).

[5] M. König, S. Wiedmann, C. Brüne, A. Roth, H. Buhmann, L. W. Molenkamp, X.-L. Qi, and S.-C. Zhang, Science 318, 766 (2007).

[6] D. Hsieh, D. Qian, L. Wray, Y. Xia, Y. S. Hor, R. J. Cava, and M. Z. Hasan, Nature (London) 452, 970 (2008).

[7] E. Prodan and C. Prodan, Phys. Rev. Lett. 103, 248101 (2009).
[8] A. B. Khanikaev, R. Fleury, S. H. Mousavi, and A. Alù, Nat. Commun. 6, 8260 (2015).

[9] C. He, X. Ni, H. Ge, X.-C. Sun, Y.-B. Chen, M.-H. Lu, X.-P. Liu, and Y.-F. Chen, Nat. Phys. 12, 1124 (2016).

[10] N. Goldman, G. Juzeliunas, P. Ohberg, and I. B. Spielman, Rep. Prog. Phys. 77, 126401 (2014).

[11] J. Yuen-Zhou, S. K. Saikin, T. Zhu, M. C. Onbasli, C. A. Ross, V. Bulovic, and M. A. Baldo, Nat. Commun. 7, 11783 (2016).

[12] D. Jin, T. Christensen, M. Soljačić, N. X. Fang, L. Lu, and X. Zhang, Phys. Rev. Lett. 118, 245301 (2017).

[13] D. Pan, R. Yu, H. Xu, and F. J. G. de Abajo, arXiv:1702.00036. 
[14] A. V. Nalitov, D. D. Solnyshkov, and G. Malpuech, Phys. Rev. Lett. 114, 116401 (2015).

[15] L. Lu, J. D. Joannopoulos, and M. Soljačić, Nat. Photon. 8, 821 (2014).

[16] L. Lu, C. Fang, L. Fu, S. G. Johnson, J. D. Joannopoulos, and M. Soljačić, Nat. Phys. 12, 337 (2016).

[17] A. Slobozhanyuk, S. H. Mousavi, X. Ni, D. Smirnova, Y. S. Kivshar, and A. B. Khanikaev, Nat. Photon. 11, 130 (2016).

[18] Z. Wang, Y. Chong, J. D. Joannopoulos, and M. Soljačić, Nature (London) 461, 772 (2009).

[19] M. C. Rechtsman, J. M. Zeuner, Y. Plotnik, Y. Lumer, D. Podolsky, F. Dreisow, S. Nolte, M. Segev, and A. Szameit, Nature (London) 496, 196 (2013).

[20] S. A. Skirlo, L. Lu, Y. Igarashi, Q. Yan, J. Joannopoulos, and M. Soljačić, Phys. Rev. Lett. 115, 253901 (2015).

[21] X. Cheng, C. Jouvaud, X. Ni, S. H. Mousavi, A. Z. Genack, and A. B. Khanikaev, Nat. Mater. 15, 542 (2016).

[22] Y. Yang, Y. F. Xu, T. Xu, H.-X. Wang, J.-H. Jiang, X. Hu, and Z. H. Hang, arXiv:1610.07780.

[23] S. Yves, R. Fleury, T. Berthelot, M. Fink, F. Lemoult, and G. Lerosey, arXiv: 1705.09225.

[24] A. B. Khanikaev, S. H. Mousavi, W.-K. Tse, M. Kargarian, A. H. MacDonald, and G. Shvets, Nat. Mater. 12, 233 (2013).

[25] N. Shitrit, I. Yulevich, E. Maguid, D. Ozeri, D. Veksler, V. Kleiner, and E. Hasman, Science 340, 724 (2013).

[26] M. Hafezi, S. Mittal, J. Fan, A. Migdall, and J. M. Taylor, Nat. Photon. 7, 1001 (2013).

[27] J.-W. Dong, X.-D. Chen, H. Zhu, Y. Wang, and X. Zhang, Nat. Mater. 16, 298 (2017).
[28] K. Y. Bliokh, F. J. Rodríguez-Fortuño, F. Nori, and A. V. Zayats, Nat. Photon. 9, 796 (2015).

[29] L. Lu, J. D. Joannopoulos, and M. Soljacic, Nat. Phys. 12, 626 (2016).

[30] L.-H. Wu and X. Hu, Phys. Rev. Lett. 114, 223901 (2015).

[31] T. Ma and G. Shvets, New J. Phys. 18, 025012 (2016).

[32] M. Milićević, T. Ozawa, G. Montambaux, I. Carusotto, E. Galopin, A. Lemaître, L. Le Gratiet, I. Sagnes, J. Bloch, and A. Amo, Phys. Rev. Lett. 118, 107403 (2017).

[33] S. Yang, Y. Wang, and H. Sun, Adv. Opt. Mater. 3, 1136 (2015).

[34] Y.Zhang, K. He, C.-Z. Chang, C.-L. Song, L.-L. Wang, X. Chen, J.-F. Jia, Z. Fang, X. Dai, W.-Y. Shan, S.-Q. Shen, Q. Niu, X.-L. Qi, S.-C. Zhang, X.-C. Ma, and Q.-K. Xue, Nat. Phys. 6, 584 (2010).

[35] B. A. Bernevig, T. L. Hughes, and S.-C. Zhang, Science 314, 1757 (2006).

[36] See Supplemental Material at http://link.aps.org/supplemental/ 10.1103/PhysRevB.96.041408 for the derivation of analytical results and the details of numerical simulations.

[37] K.-I. Imura, Y. Takane, and A. Tanaka, Phys. Rev. B 84, 195406 (2011).

[38] K.-I. Imura, Y. Yoshimura, Y. Takane, and T. Fukui, Phys. Rev. B 86, 235119 (2012).

[39] G. Siroki, D. K. K. Lee, P. D. Haynes, and V. Giannini, Nat. Commun. 7, 12375 (2016).

[40] S. G. Johnson and J. D. Joannopoulos, Opt. Express 8, 173 (2001).

[41] A. F. Oskooi, D. Roundy, M. Ibanescu, P. Bermel, J. D. Joannopoulos, and S. G. Johnson, Comput. Phys. Commun. 181, 687 (2010). 